\section{Scattering of X-Rays by Bound Electrons.}

Thovgh Bergen Davis and his collaborators (Phys. Rev., 23) have reported the detection by means of an ionisation chamber of the modified lines (over and above the Compton effect) produced by the scattering of a monochromatic beam of X-rays by carbon in a direction at right angles to the direction of propagation, Coster (Nature, Aug. 10, 1929), Ehrenberg (Zeit. $f$. Phys., 53), and Kast (Zeit. f. Phys., 58) have failed to observe the same on the photographic plate. In a previous note to NATURF, it has been pointed out

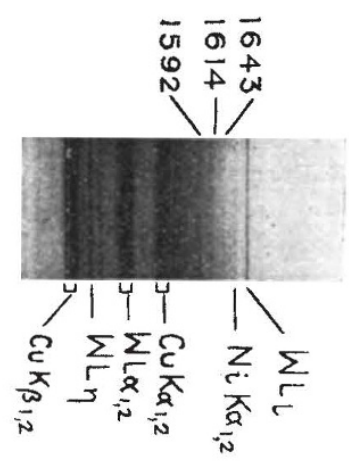

FIG. 1. by me that a portion of copper $K a$ radiation in passing through carbon decreases its frequency by an amount equal to that of the $K a$ radiation of carbon. The accompanying photograph (Fig. 1) shows the effect of copper $K a$ radiation in passing through carbon, nitrogen, and oxygen. The known lines are marked as usual, while the new ones are shown by numbers. The white mark on the copper $K a$ line is due to the fact that a piece of aluminium was placed just

in front of the photographic plate to decrease the blackness of the plate in this region.

The lines are diffuse and very broad, and as such they could only be measured with a glass scale. Longer exposure does not improve the relative intensity of these lines, as the general radiation tends to blacken the whole plate. The three lines $1592 \mathrm{X} . \mathrm{U}$., $1614 \mathrm{X} . \mathrm{U}$., and $1643 \mathrm{X} . \mathrm{U}$. are interpreted as due to the scattering of copper $K a$ radiation by carbon, nitrogen, and oxygen. The frequency differences $(\nu / R)$ between the copper $K a$ radiation and these lines are $20 \cdot 1,27 \cdot 6$, and $37 \cdot 7$ respectively, whereas the frequencies $(\nu / R)$ of the $K \alpha$ radiations of carbon, nitrogen, and oxygen are $20 \cdot 4,28 \cdot 7$, and $38 \cdot 3$ respectively.

Similarly, a portion of the nickel $K a$ radiation in passing through carbon and nitrogen shows new lines the wave-lengths of which are 1719 X.U. and 1746 $X . U$., and the frequency differences between the nickel $K a$ radiation and these lines are 20.0 and 28.1 respectively.

The absence of any modified lines by scattering (Coster, Ehrenberg, and Kast) in any other direction than that of propagation suggests that in all probability in an interaction between a quantum and a bound electron, the former on its passage through the medium loses a part of its energy and an equivalent amount of momentum in raising the electron from one orbit to the other, and is propagated in the original direction as a modified wave of lower frequency.

Department of Physics,

University College of Science,

92 Upper Circular Road, Caleutta, April 23.

\section{Fluorescence of Mercury Vapour in the Far U1tra-Violet.}

Some years ago one of us (Terenin : Zeits.f. Physik, 31 , p. $40 ; 1925$ ) observed a strong re-emission of the aluminium line at $1854 \mathrm{~A}$. in the fluorescent spectrum of dense mercury vapour excited by an aluminium spark. A great deal of work has been done since on mercury band fluorescence, but this re-emission has not been recorded again. This can be explained by the strong absorption of the radiation in ordinary spectrographs with thick quartz and a long light-path in air ; a very small home-made fluorite spectrograph was used in the experiment mentioned. Last year this subject was re-examined by us in more detail. The part played by absorption was shown clearly by the fact that an exposure of five hours was needed to record the emission with an ordinary small quartz spectrograph, whereas five minutes sufficed with the fluorite one. In spite of the short focal length of the lenses $(5 \mathrm{~cm}$.) the dispersion of the fluorite prism was sufficient to show the aluminium lines at I854 A. and 1862 A. clearly resolved.

Re-emission of the $1854 \mathrm{~A}$. line begins to be noticeable at a much smaller vapour density than is required for mercury band fluorescence in the nearer ultra-violet and the visible. At a vapour pressure of about $10 \mathrm{~mm}$., the re-emission is quite conspicuous. On raising the pressure of the vapour, the aluminium lines at $1862 \mathrm{~A} ., 1935 \mathrm{~A}$., and $1990 \mathrm{~A}$. appear in succession, following the development of the well-known continuous absorption band to the long wave-length side of $1850 \mathrm{~A}$. There is in addition in the fluorescent spectrum a continuous background, which spreads with increase in vapour density from $1854 \mathrm{~A}$. to 2345 A., where it ceases abruptly. A similar emission, likewise localised in the neighbourhood of the resonance line $1^{1} S_{0}-2^{1} P_{1}$ of the atom, has been observed in the case of cadmium by Kapuscinski (Zeits. $f$. Physik, 41, p. 214 ; 1927). The remarkable re-emission of exciting lines is no doubt of molecular origin, but its exact mechanism is still not clear.

On heating the vapour to $800^{\circ} \mathrm{C}$. at constant pressure, the spectrum described above is quenched as a whole, which suggests a common origin for the reemitted lines and the continuous background.

The presence of small traces of gases does not have the strong quenching effect which occurs with the near ultra-violet and visible fluorescence. This is consistent with the view that the state to which the molecule $\mathrm{Hg}_{2}$ is raised by the absorption of the aluminium lines has a much shorter life than those responsible for the fluorescence in the near ultraviolet and visible; the duration of the latter has been shown to be of the order of $10^{-3} \mathrm{sec}$. (Rayleigh, Proc. Roy. Soc., 114, 620; 1927. Pringsheim and Terenin, Zeits. f. Physik, 47, p. $330 ; 1928)$. It is of interest that the band at $2345 \mathrm{~A}$. (probably corresponding to the $2^{3} P_{2}$ state of mercury) is strongly quenched by traces of gases, and so does not seem to be connected with the far ultra-violet fluorescence studied here.

A quantitative investigation of the subject is in progress.

M. Eliashevich.

Physical Institute,

A. Terenin.

University of Leningrad, May 1.

The Acquired Gharacters of Alytes.

In NATURE of April 12, p. 562, Dr. Charles Walker draws a parallel between the well-known experiments on plants transferred to other climate or soil and Kammerer's endeavours to prove the inheritance of acquired characteristics, especially in Alytesobstetricans. Without again entering into the whole matter, I would only like to point out that Kammerer (Roux' Archiv, 45,$324 ; 1919$ ) states distinctly that the Alytes returned to their habitual temperature retained the habit acquired at the high temperature artificially employed of copulating in the water (p. 328), and that the fourth generation of Alytes which were returned to normal conditions developed the nuptial pad in a male of the $F_{6}$-generation. It is the habit which was

No. 3162 , VoL. 125] 